\title{
Cordial Volterra Integral Equations with Vanishing Delays*
}

\author{
Hongjiu Wang1,2\#, Zhanwen Yang2,3, Melusi Khumalo² \\ ${ }^{1}$ College of Science, Heilongjiang University of Science and Technology, Harbin, China \\ ${ }^{2}$ Department of Pure and Applied Mathematics, University of Johannesburg, Auckland Park, Johannesburg, South Africa \\ ${ }^{3}$ Science Research Center, The Academy of Fundamental and Interdisciplinary Science, Harbin Institute of Technology, Harbin, \\ China \\ Email: "whj1980329@163.com
}

How to cite this paper: Wang, H.J., Yang, Z.W. and Khumalo, M. (2017) Cordial Volterra Integral Equations with Vanishing Delays. Journal of Applied Mathematics and Physics, 5, 294-302.

https://doi.org/10.4236/jamp.2017.52026

Received: October 3, 2016

Accepted: February 12, 2017

Published: February 15, 2017

\begin{abstract}
Cordial Volterra integral equations (CVIEs) from some applications models associated with a noncompact cordial Volterra integral operator are discussed in the recent years. A lot of real problems are effected by a delayed history information. In this paper we investigate some properties of cordial Volterra integral operators influenced by a vanishing delay. It is shown that to replicate all eigenfunctions $t^{\lambda}, \lambda=0$ or $\Re \lambda>0$, the vanishing delay must be a proportional delay. For such a linear delay, the spectrum, eigenvalues and eigenfunctions of the operators and the existence, uniqueness and solution spaces of solutions are presented. For a nonlinear vanishing delay, we show a necessary and sufficient condition such that the operator is compact, which also yields the existence and uniqueness of solutions to CVIEs with the vanishing delay.
\end{abstract}

\section{Keywords}

Cordial Volterra Integral Equations, Vanishing Delay, Propositional Delay, Compactness, Existence and Uniqueness

\section{Introduction}

A kind of Volterra integral equations with weakly singular kernels arisen in 1975 [1] from some heat condition problems with mixed-type boundary conditions is transformed by Watson transforms [2] and the convolution theorem [3]. In [4], the author generalizes such kind of equations into cordial Volterra integral equations (CVIEs) with the form

${ }^{*}$ This work is supported by the Natural Science Foundation of Heilongjiang Province, China (Grant No. QC2013C019). 


$$
\mu u(t)=f(t)+\left(\mathcal{V}_{\varphi} u\right)(t), \quad t \in I:=[0, T],
$$

where $T>0$, the core $\varphi \in L^{1}(0,1)$ and the cordial Volterra integral operator is defined by

$$
\left(\mathcal{V}_{\varphi} u\right)(t):=\int_{0}^{t} t^{-1} \varphi(s / t) u(s) \mathrm{d} s=\int_{0}^{1} \varphi(x) u(t x) \mathrm{d} x .
$$

CVIEs appear in a lot of application models, such as Diogo core $\varphi(x)=\left(1-x^{2}\right)^{-\frac{1}{2}}$, linear Lighthill's equation $\left(\varphi(x)=\left(1-x^{\frac{3}{2}}\right)^{-\frac{2}{3}}\right)$, and so on.

It is shown that the cordial Volterra integral operator $\mathcal{V}_{\varphi}$ in the Banach space $C(I)$ is noncompact and its spectrum is a non-countable set, i.e.,

$$
\sigma\left(\mathcal{V}_{\varphi}\right)=\{0\} \cup\{\hat{\varphi}(\lambda): \Re \lambda \geq 0\},
$$

where

$$
\hat{\varphi}(\lambda)=\int_{0}^{1} \varphi(x) x^{\lambda} \mathrm{d} x .
$$

In [5], the author describes the eigenvalues and eigenfucntions of the operator $\mathcal{V}_{\varphi}$ on the space $C(I)$ when $\varphi \in L^{p}(0,1)$ with some $p>1$ :

1) the point spectrum of $\mathcal{V}_{\varphi}$ is exactly the set $\sigma^{+}\left(\mathcal{V}_{\varphi}\right)=\{\hat{\varphi}(\lambda): \Re \lambda>0\}$;

2) the dimension of the null space $\mathcal{N}\left(\mu \mathcal{I}-\mathcal{V}_{\varphi}\right)$ is the sum of the multiplicities of the roots of $\gamma_{\mu}(\lambda):=\mu-\hat{\varphi}(\lambda)=0$ in the complex plane

$\{\Re \lambda>0: \lambda \in \mathbb{C}\}$;

3) the linearly independent eigenfunctions are given by

$$
t^{\lambda}, \ldots, t^{\lambda}(\ln t)^{k^{\lambda}},
$$

where $k_{\lambda}$ is the multiplicity of the root $\lambda$ of $\gamma_{\mu}(\lambda)=0$.

The pure Volterra integral equations with vanishing delay (VIEwND) are initially studied in [6] and a special form of VIEwND, proportional delay differential equations, is widely used in practical applications, for example, electrodynamics [7] [8], nonlinear dynamical systems [9] [10], and also the survey papers [11] [12]. In this paper, we consider the CVIEs with a vanishing delay,

$$
\mu u(t)=f(t)+\left(\mathcal{V}_{\theta, \varphi} u\right)(t), t \in I,
$$

where $\theta(t)$ is a continuous delay function such that $\theta(0)=0$ and $\theta(t)<t$ for all $0<t \in I$ and the operator with delay is similarly defined by

$$
\left(\mathcal{V}_{\theta, \varphi} u\right)(t):=\int_{\theta(t)}^{t} t^{-1} \varphi(s / t) u(s) \mathrm{d} s .
$$

Besides the existence and uniqueness of solutions to (2), it is more interesting how the eigenvalues and eigenfunctions of the operators are influenced by vanishing delays. In Section 2, we show that the proportional delay $\theta(t)=q t$, $0<q<1$, is the only one that replicates all eigenfunctions $t^{\lambda}, \lambda=0$ or $\Re \lambda>0$. For such a delay, we describe the spectrum, eigenvalues and eigenfunctions of the operator $\mathcal{V}_{\theta, \varphi}$. In Section 3, we present a necessary and sufficient condition for the compactness of the operator $\mathcal{V}_{\theta, \varphi}$ with a vanishing delay. Based on these discussions, we present the existence, uniqueness and the construction 
of solutions to (2).

\section{Propositional Delays}

For a vanishing delay $\theta(t)$ satisfying that

(D1) $\theta(0)=0$,

(D2) $0 \leq \theta(t)<t$ for all $0<t \in I$,

(D3) $\theta(t)$ is a continuous function in the interval $I$ and $\theta^{\prime}(0)$ exists, the operator (3) is rewritten as the following form

$$
\left(\mathcal{V}_{\theta, \varphi} u\right)(t)=\left(\mathcal{W}_{\xi, \varphi} u\right)(t):=\int_{\xi(t)}^{1} \varphi(x) u(t x) \mathrm{d} x,
$$

where the function $\xi(t)=\frac{\theta(t)}{t}$ is a well-defined continuous function in the whole interval $I$. Obviously

$$
0 \leq \xi(0)=\lim _{t \rightarrow 0+} \xi(t)=\theta^{\prime}(0) \leq 1,
$$

and $\xi(t) \in[0,1]$ for all $t \in I$.

The cordial Volterra integral operator with a vanishing delay (3) is also written as a cordial Volterra integral operator with a variable kernel, i.e.,

$$
\left(\mathcal{V}_{\theta, \varphi} u\right)(t)=\left(\mathcal{V}_{\varphi, a} u\right)(t):=\int_{0}^{1} \varphi(x) a(t, t x) u(t x) \mathrm{d} x,
$$

where the discontinuous kernel $a(t, s)$ is defined by

$$
a(t, s)= \begin{cases}0, & 0 \leq s \leq \theta(t), \\ 1, & \theta(t)<s \leq t .\end{cases}
$$

The properties of the operator $\mathcal{V}_{\varphi, a}$ with continuous kernels are investigated in [13] such as it is compact if and only if $a(0,0)=0$. From the above definition, the discontinuous function $a$ always satisfies $a(0,0)=0$, but the compactness of the operator $\mathcal{V}_{\theta, \varphi}$ is influenced not only by the core but also by the value of $\theta^{\prime}(0)$ (see in Corollary 2.3 and Theorem 3.1).

Theorem 2.1. Assume that the function $\xi \in C(I)$.

1) The operator $\mathcal{V}_{\theta, \varphi}$ is a bounded operator from $C(I)$ to $C(I)$.

2) If all power-functions $t^{\lambda}, \lambda=0$ or $\mathfrak{R} \lambda>0$, are eigenfunctions of $\mathcal{V}_{\theta, \varphi}$, then

$$
\int_{\xi(0)}^{\xi(t)}|\varphi(x)| \mathrm{d} x \equiv 0
$$

where for $\xi(t)<\xi(0)$, the integration is defined by

$$
\int_{\xi(0)}^{\xi(t)}|\varphi(x)| \mathrm{d} x=-\int_{\xi(t)}^{\xi(0)}|\varphi(x)| \mathrm{d} x .
$$

Proof. (i) For $\varphi \in L^{1}(0,1), u \in C(I)$ and $\epsilon>0$, there exists a $\delta_{1}=\delta_{1}(\epsilon, u)>0$ such that

$$
\int_{a}^{\min \left\{1, a+\delta_{1}\right\}}|\varphi(x)| \mathrm{d} x<\frac{1}{2\left(1+\|u\|_{\infty}\right)} \epsilon \text { for all } a \in[0,1]
$$

and for all $s_{1}, s_{2} \in I$ with $\left|s_{1}-s_{2}\right| \leq \delta_{1}$

$$
\left|u\left(s_{1}\right)-u\left(s_{2}\right)\right| \leq \frac{1}{2\left(1+\|\varphi\|_{1}\right)} \epsilon,
$$


since $u$ is uniformly continuous on the closed interval. The uniform continuity of $\xi$ implies that there exists a $\delta \leq \delta_{1}$ such that $\left|\xi\left(t_{1}\right)-\xi\left(t_{2}\right)\right| \leq \delta_{1}$ for all $t_{1}, t_{2} \in I$ with $\left|t_{1}-t_{2}\right| \leq \delta$.

We, without loss of generality, assume that $\xi\left(t_{1}\right) \geq \xi\left(t_{2}\right)$ in the following estimation. Then

$$
\begin{aligned}
& \left|\left(\mathcal{V}_{\theta, \varphi} u\right)\left(t_{1}\right)-\left(\mathcal{V}_{\theta, \varphi} u\right)\left(t_{2}\right)\right|=\left|\left(\mathcal{W}_{\xi, \varphi} u\right)\left(t_{1}\right)-\left(\mathcal{W}_{\xi, \varphi} u\right)\left(t_{2}\right)\right| \\
& \leq \int_{\xi\left(t_{1}\right)}^{\xi\left(t_{2}\right)}|\varphi(x)|\left|u\left(t_{1} x\right)\right| \mathrm{d} x+\int_{\xi\left(t_{2}\right)}^{1}|\varphi(x)|\left|u\left(t_{1} x\right)-u\left(t_{2} x\right)\right| \mathrm{d} x \\
& \leq\|u\|_{\infty} \int_{\xi\left(t_{1}\right)}^{\min \left\{1, \xi\left(t_{1}\right)+\delta_{1}\right\}}|\varphi(x)| \mathrm{d} x+\frac{1}{2\left(1+\|\varphi\|_{1}\right)} \epsilon \int_{0}^{1}|\varphi(x)| \mathrm{d} x \leq \epsilon .
\end{aligned}
$$

Hence $\mathcal{V}_{\theta, \varphi}$ maps $C(I)$ to $C(I)$ and its boundedness comes from

$$
\begin{aligned}
\max _{t \in[0, T]}\left|\left(\mathcal{V}_{\theta, \varphi} u\right)(t)\right| & =\max _{t \in[0, T]}\left|\left(\mathcal{W}_{\xi, \varphi} u\right)(t)\right| \\
& \leq \max _{t \in[0, T]}\left|\int_{\xi(t)}^{1} \varphi(x) u(t x) \mathrm{d} x\right| \leq\|\varphi\|_{1}\|u\|_{\infty} .
\end{aligned}
$$

2) Without loss of generality, suppose that $\xi\left(t^{*}\right)>\xi(0)$ and

$\int_{\xi(0)}^{\xi\left(t^{*}\right)}|\varphi(x)| \mathrm{d} x>0$ for some $t^{*} \in(0, T]$. Then similarly to the approach in [4], there exists a polynomial $p(t)=\sum_{i=0}^{n} p_{i} t^{i}$ such that

$$
\int_{\xi(0)}^{\xi\left(t^{*}\right)} \varphi(x) p\left(t^{*} x\right) \mathrm{d} x \geq \frac{1}{2} \int_{\xi(0)}^{\xi\left(t^{*}\right)}|\varphi(x)| \mathrm{d} x>0
$$

Since $t^{i}, i=0,1, \ldots$, is an eigenfunction of $\mathcal{W}_{\xi, \varphi}$,

$$
\int_{\xi(t)}^{1} \varphi(x) x^{i} \mathrm{~d} x=c_{i}
$$

is also independent of $t$ for $i=0,1, \ldots$ Thus

$$
\begin{aligned}
& \sum_{i=0}^{n} \int_{\xi(0)}^{1} \varphi(x) p_{i}\left(t^{*} x\right)^{i} \mathrm{~d} x=\sum_{i=0}^{n} p_{i}\left(t^{*}\right)^{i} \int_{\xi(0)}^{1} \varphi(x) x^{i} \mathrm{~d} x=\sum_{i=0}^{n} p_{i} c_{i}\left(t^{*}\right)^{i}, \\
& \sum_{i=0}^{n} \int_{\xi\left(t^{*}\right)}^{1} \varphi(x) p_{i}\left(t^{*} x\right)^{i} \mathrm{~d} x=\sum_{i=0}^{n} p_{i}\left(t^{*}\right)^{i} \int_{\xi\left(t^{*}\right)}^{1} \varphi(x) x^{i} \mathrm{~d} x=\sum_{i=0}^{n} p_{i} c_{i}\left(t^{*}\right)^{i},
\end{aligned}
$$

and hence

$$
\int_{\xi(0)}^{\xi\left(t^{*}\right)} \varphi(x) p\left(t^{*} x\right) \mathrm{d} x=0 .
$$

This contradiction implies the proof is complete.

Remark 2.2. In [4], the author shows that an operator $\mathcal{K}$ mapping $C(I)$ to $C(I)$ has the two properties:

1) $\mathcal{K}$ is a bounded operator;

2) all power-functions $t^{\lambda}, \lambda=0$ or $\mathfrak{R} \lambda>0$, are eigenfunctions of $\mathcal{K}$; if and only if $\mathcal{K}=\mathcal{V}_{\varphi}$ is a cordial Volterra integral operator. While including vanishing delays, the two properties only hold for a proportional delay $\theta(t)=q t, \quad 0<q<1$.

For a core $\varphi \in L^{1}(0,1)$, we define an integration function of the core by

$$
\Phi(x)=\int_{0}^{x}|\varphi(r)| \mathrm{d} r .
$$


If $\Phi(x) \equiv 0$ for $x \in[0, q]$ with some $0<q<1$ (or $\operatorname{supp} \varphi \in[q, 1]$ ), then CVIEs naturally reduce to a proportional delay form

$$
\mu u(t)=f(t)+\left(\mathcal{W}_{q, \varphi} u\right)(t),
$$

where the corresponding operator has the form $\mathcal{W}_{q, \varphi}=\mathcal{V}_{\varphi_{q}}$ with $\varphi_{q}(x):=\mathcal{X}_{q}(x) \varphi(x)$ and

$$
\mathcal{X}_{q}(x):= \begin{cases}0, & x \in[0, q), \\ 1, & x \in[q, 1] .\end{cases}
$$

Corollary 2.3. Assume that $\varphi \in L^{1}(0,1)$ and $\Phi(x)$ is a strictly increasing function for $x \in[0,1]$. Then a cordial Volterra integral operator with vanishing delays opposites the two properties in Remark 2.2 if and only if the delay $\theta(t)=q t$ is a proportional delay. Of course it is a noncompact operator.

Proof. By Theorem 2.1, one obtains that $\xi(t) \equiv \xi(0)$ is a constant. Thus the proof is completed by $\theta(t)=t \xi(t)$.

Based on $\mathcal{W}_{q, \varphi}=\mathcal{V}_{\varphi_{q}}$, some more detailed properties on cordial Volterra integral operators with a proportional delay are presented in the following theorem.

Theorem 2.4. Assume that a core $\varphi \in L^{p}(0,1)$ with some $p>1, \theta(t)=q t$, $0<q<1$ and $\Phi(x)>0$ for $x \in(q, 1]$. Then

1) The spectrum of $\mathcal{V}_{\theta, \varphi}$ is given by

$$
\sigma\left(\mathcal{V}_{\theta, \varphi}\right)=\{0\} \cup\left\{\hat{\varphi}_{q}(\lambda): \Re \lambda \geq 0\right\},
$$

where $\hat{\varphi}_{q}(\lambda):=\int_{0}^{1} \varphi_{q}(x) x^{\lambda} \mathrm{d} x=\int_{q}^{1} \varphi(x) x^{\lambda} \mathrm{d} x$.

2) The point spectrum of $\mathcal{V}_{\theta, \varphi}$ is exactly the set $\sigma^{+}\left(\mathcal{V}_{\theta, \varphi}\right)=\left\{\hat{\varphi}_{q}(\lambda): \Re \lambda>0\right\}$.

3) The dimension of the null space $\mathcal{N}\left(\mu \mathcal{I}-\mathcal{V}_{\theta, \varphi}\right)$ is the sum of the multiplicities of the roots of $\gamma_{q, \mu}(\lambda):=\mu-\hat{\varphi}_{q}(\lambda)=0$ in the complex plane $\{\mathfrak{R} \lambda>0: \lambda \in \mathbb{C}\}$.

4) The linearly independent eigenfunctions are given by

$$
t^{\lambda}, \ldots, t^{\lambda}(\ln t)^{k_{\lambda}}
$$

where $k_{\lambda}$ is the multiplicity of the root $\lambda$ of $\gamma_{q, \mu}(\lambda)=0$.

5) The range of the operator $\mu \mathcal{I}-\mathcal{V}_{\theta, \varphi}$ is the whole space $C(I)$ if and only if $\mu \notin \sigma^{0}\left(\mathcal{V}_{\theta, \varphi}\right)=\left\{\hat{\varphi}_{q}(\lambda): \Re \lambda=0\right\} \cup\{0\}$.

Both the existence and uniqueness of solutions to (5) are valid when the parameter $\mu$ does not lie in the spectrum of the corresponding operators. On the other hand, for $\mu$ lying in the spectrum, by the same technique in [5], we are also able to construct solutions to (5). For convenience, we review some notations in [5]:

1) $C_{c_{1}, \ldots, c_{n}}[0, T]=\left\{u \in C[0, T]: u\left(c_{i}\right)=0, i=1, \ldots, n\right\}$ with different parameters $c_{1}, \ldots, c_{n} \in(0, T]$

2) $C^{\epsilon}[0, T]:=\left\{t^{\epsilon} w(t): w \in C[0, T]\right\}$ with the norm

$$
\|u\|_{\epsilon}:=\|w\| \text { for } u \in C^{\epsilon}[0, T] \text {. }
$$

Theorem 2.5. Assume that $\varphi \in L^{p}(0,1)$ with some $p>1$ and that $\theta(t)=q t$, $0<q<1$, and $\Phi(x)>0$ for $x \in(q, 1]$. Let $n=\operatorname{dim} \mathcal{N}\left(\mu \mathcal{I}-\mathcal{V}_{\theta, \varphi}\right)$. Then there 
exist $c_{1}, \ldots, c_{n}$ distinct points in $(0, T]$ such that the following statements are true.

1) For $\mu \in \sigma^{+}\left(\mathcal{V}_{\theta, \varphi}\right) \backslash \sigma^{0}\left(\mathcal{V}_{\theta, \varphi}\right)$, there exists a unique solution $u^{*} \in C_{c_{1}, \ldots, c_{n}}[0, T]$ to (5) that continuously depends on $f \in C[0, T]$, and all solutions have the form

$$
u=u^{*}+u^{\perp},
$$

where $u^{\perp}$ is a linear combination of functions fo functions $t^{\lambda^{*}}(\ln t)^{i}$, $i=0, \ldots, k_{\lambda^{*}}-1$, and $\lambda^{*} \in \mathbb{C}^{+}$is a root of $\gamma_{q, \mu}(\lambda)=0$ with multiplicity $k_{\lambda^{*}}$.

2) For $\mu \in \sigma^{0}\left(\mathcal{V}_{\theta, \varphi}\right) \backslash \sigma^{+}\left(\mathcal{V}_{\theta, \varphi}\right)$, there exists at most one solution to (5), and there exists exactly one solution to (5) when $f \in C^{\epsilon}[0, T]$ for any $\epsilon>0$.

3) For $\mu \in \sigma^{0}\left(\mathcal{V}_{\theta, \varphi}\right) \cap \sigma^{+}\left(\mathcal{V}_{\theta, \varphi}\right)$, there exists at most one solution $u^{*}$ belonging to $C_{c_{1}, \ldots, c_{n}}[0, T]$, and there exists a unique solution in $u^{*} \in C_{c_{1}, \ldots, c_{n}}[0, T]$ for any $\epsilon>0$ and $f \in C^{\epsilon}[0, T]$. All solutions have the form

$$
u=u^{*}+u^{\perp},
$$

where $u^{\perp}$ is linearly combined by such functions 1 (if $\left.\mu=\hat{\varphi}_{q}(0)\right)$ and

$t^{\lambda^{*}}(\ln t)^{i}, \quad i=0,1, \ldots, k_{\lambda^{*}}-1$, and $\lambda^{*} \in \mathbb{C}^{+}$is a root of $\gamma_{q, \mu}(\lambda)=0$ with multiplicity $k_{\lambda^{*}}$.

\section{General Vanishing Delays}

For a more general vanishing delay, the compactness of the cordial Volterra integral operators is influenced by the value of $\theta^{\prime}(0)$.

Theorem 3.1. Assume that $\varphi \in L^{1}(0,1)$ and that the delay function $\theta(t)$ satisfies the assumptions $(D 1),(D 2),(D 3)$. Then the operator $\mathcal{V}_{\theta, \varphi}$ is compact in $C(I)$ if and only if supp $\varphi \subseteq\left[0, \theta^{\prime}(0)\right]$.

Proof. From the definition of the function $\xi$, it is known that $\xi(0)=\theta^{\prime}(0)$. In Lemma 3.6, one obtains from supp $\varphi \subseteq\left[0, \theta^{\prime}(0)\right]$ that $\left(\mathcal{V}_{\theta, \varphi} u\right)(0)=0$ for all $u \in C(I)$. Hence by Ascoli-Arzela theorem, the compactness of the cordial Volterra integral operator $\mathcal{V}_{\theta, \varphi}$ with such a vanishing delay term is shown in Lemma 3.7. The proof will be completed, when the non-compactness of the operator is proved in Lemma 3.8.

The simplest compact condition according to Theorem 3.1 is $\theta^{\prime}(0)=1$.

Corollary 3.2. Assume that $\varphi \in L^{1}(0,1)$ and that the delay function $\theta(t)$ satisfies the assumptions (D1), (D2), (D3). Then the operator $\mathcal{V}_{\theta, \varphi}$ is compact in $C(I)$ for any core $\varphi \in L^{1}(0,1)$ provided that $\theta^{\prime}(0)=1$.

Remark 3.3. Consider the constant core $\varphi(x) \equiv 1$. Then

1) $\mathcal{V}_{q, 1}, 0<q<1$, are non-compact in $C(I)$.

2) For $\theta(t)=\sin t, \mathcal{V}_{\theta, 1}$ is compact in $C([0,1])$.

The existence and uniqueness of solutions to (2) is similar to the classical second kind of VIEs when the corresponding operator is compact.

Theorem 3.4. Assume that $\varphi \in L^{1}(0,1)$ and that the delay function $\theta(t)$ satisfies the assumptions $(D 1),(D 2),(D 3)$ and that supp $\varphi \subseteq\left[0, \theta^{\prime}(0)\right]$. Then for all $\mu \neq 0$ and all $f \in C(I)$, there exists a unique solution to (2).

Proof. In Lemma 3.9, it is shown that the null space of the operator $\mu \mathcal{I}-\mathcal{V}_{\theta, \varphi}$ 
in $C(I)$ is $\{0\}$, which together with the compactness of $\mathcal{V}_{\theta, \varphi}$ implies that the operator $\mu \mathcal{I}-\mathcal{V}_{\theta, \varphi}$ has a bounded inverse in $C(I)$ (see in [14]). Hence the proof is complete.

Example 3.5. Consider the following CVIEs with a vanishing delay

1) $\varphi(x)=\left(\frac{1}{2}-x\right)_{+}$and $\theta(t)=\frac{1}{2} t-t^{2}$ for $t \in I=\left[0, \frac{1}{2}\right]$;

2) $\varphi(x)=\left(1-x^{\frac{3}{2}}\right)^{-\frac{2}{3}}$ (the linear form of Lighthill's equations) and $\theta(t)=\sin t$ for $t \in I=[0,1]$;

3) $\varphi(x)=\left(\frac{1}{2}-x\right)_{+}$and $\theta(t)=\frac{1}{2} t+t^{2}$ for $t \in I=\left[0, \frac{1}{2}\right]$.

Then the corresponding operators are compact and there exists a unique solution to (2) for $\mu \neq 0$ and $f \in C(I)$.

Theorems 3.1 and 3.4 are proved by the following lemmas.

Lemma 3.6 Assume that $\varphi \in L^{1}(0,1)$ and that $\xi(t) \in[0,1]$ is a continuous function in $I$. Then one obtains that $\left(\mathcal{V}_{\theta, \varphi} u\right)(0)=0$ for all $u \in C(I)$ if supp $\varphi \subseteq[0, \xi(0)]$.

Proof. In view of

$$
\left(\mathcal{V}_{\theta, \varphi} u\right)(0)=\left(\mathcal{W}_{\xi, \varphi} u\right)(0)=u(0) \int_{\xi(0)}^{1} \varphi(x) \mathrm{d} x,
$$

the condition in this lemma yields that for all $u \in C(I)$,

$$
\left(\mathcal{V}_{\theta, \varphi} u\right)(0)=0
$$

The proof is complete.

Lemma 3.7 Assume that $\varphi \in L^{1}(0,1), \xi(t) \in[0,1]$ is a continuous function in I and that supp $\varphi \subseteq[0, \xi(0)]$. Then $\mathcal{W}_{\xi, \varphi}$ is a compact operator in $C(I)$.

Proof. By Ascoli-Arzela theorem, the compactness will be proved by the equiv-continuity of $\left(\mathcal{W}_{\xi, \varphi} u\right)(t)$

Since $\xi(t)$ is a continuous function of $t$ and $\Phi(x)$ is a continuous function of $x$, for any given $\epsilon>0$ there exists an $T^{*}(\epsilon)>0$ such that

$$
|\Phi(\xi(t))-\Phi(\xi(0))| \leq \frac{1}{2} \epsilon \text { for } t \in\left[0, T^{*}(\epsilon)\right] \text {. }
$$

Therefore, for $u \in C(I)$ with $\|u\|_{\infty}=1, \quad\left(\mathcal{W}_{\xi, \varphi} u\right)(0)=0$ by Lemma 3.6 and for $t \in\left[0, T^{*}(\epsilon)\right]$,

$$
\left|\left(\mathcal{W}_{\xi, \varphi} u\right)(t)\right| \leq|\Phi(\xi(t))-\Phi(\xi(0))| \leq \frac{1}{2} \epsilon .
$$

In the following, we let $\frac{1}{2} T^{*} \leq t_{1}<t_{2} \leq T$ and we choose $\delta=\delta(\epsilon)>0$ such that for all $\left|t_{2}-t_{1}\right| \leq \delta$ implies

$$
\begin{aligned}
& \left|\Phi\left(\xi\left(t_{1}\right)\right)-\Phi\left(\xi\left(t_{2}\right)\right)\right| \leq \frac{1}{3} \epsilon, \\
& \int_{0}^{1}\left|\varphi\left(\frac{t_{1}}{t_{2}} x\right)-\varphi(x)\right| \mathrm{d} x \leq \frac{1}{3} \epsilon, \\
& \frac{2\left|t_{2}-t_{1}\right|}{T^{*}}\|\varphi\|_{1} \leq \frac{1}{3} \epsilon .
\end{aligned}
$$


Therefore,

$$
\begin{aligned}
& \left|\left(\mathcal{W}_{\xi, \varphi} u\right)\left(t_{2}\right)-\left(\mathcal{W}_{\xi, \varphi} u\right)\left(t_{1}\right)\right| \\
& \leq\left|\int_{\xi\left(t_{1}\right)}^{\xi\left(t_{2}\right)}\right| \varphi(x)|\mathrm{d} x|+\int_{0}^{1}|\varphi(x)|\left|u\left(t_{2} x\right)-u\left(t_{1} x\right)\right| \mathrm{d} x \\
& \leq \frac{1}{3} \epsilon+\left|\frac{t_{1}}{t_{2}}-1\right|\|\varphi\|_{1}+\int_{0}^{1}\left|\varphi\left(\frac{t_{1}}{t_{2}} x\right)-\varphi(x)\right| \mathrm{d} x \leq \epsilon .
\end{aligned}
$$

The proof is complete.

Lemma 3.8. Assume that $\varphi \in L^{1}(0,1), \xi(0)<1$ and that $\Phi(\xi(0))<\Phi(1)$. Then $\mathcal{W}_{\xi, \varphi}$ is a noncompact operator in $C(I)$.

Proof. Without loss of generality, we assume that $\xi(t) \geq \xi(0) \quad$ (or $\xi(t) \leq \xi(0))$ for all $t \in I$ and suppose that the operator $\mathcal{W}_{\xi, \varphi}$ is compact. Then the operator

$$
\left(\mathcal{W}_{\xi(0), \varphi} u\right)(t)=\left(\mathcal{W}_{\xi, \varphi} u\right)(t)+\int_{\xi(0)}^{\xi(t)} \varphi(x) u(t x) \mathrm{d} x
$$

or

$$
\left(\mathcal{W}_{\xi(0), \varphi} u\right)(t)=\left(\mathcal{W}_{\xi, \varphi} u\right)(t)-\int_{\xi(t)}^{\xi(0)} \varphi(x) u(t x) \mathrm{d} x
$$

is compact by Lemma 3.7. This contradicts to Corollary 2.3 and the proof is complete.

Lemma 3.9 Assume that $\varphi \in L^{1}(0,1), \xi(t) \in[0,1]$ is a continuous function in $I$ and that supp $\varphi \subseteq\left[0, \theta^{\prime}(0)\right]$. Then the null space of $\mu \mathcal{I}-\mathcal{W}_{\xi, \varphi}$ is trivial in $C(I)$ for all $\mu \neq 0$.

Proof. We suppose that $\mu \neq 0$ and there exists a $u \in C(I)$ such that

$$
\mathcal{W}_{\xi, \varphi} u=\mu u \text {. }
$$

Then $\left.u\right|_{\left[0, T^{*}(|\mu|)\right]} \equiv 0$ by

$$
\left|\left(\mathcal{W}_{\xi, \varphi} u\right)(t)\right| \leq \frac{1}{2}|\mu|\|u\|_{\infty,\left[0, T^{*}(|\mu|)\right]} .
$$

Thus, (6) reduces to

$$
\mu u(t)=\int_{\xi(t)}^{1} \varphi(x) u(t x) \mathrm{d} x, \quad t>T^{*}(|\mu|) .
$$

For all $\lambda<0$ and $\delta>0$, it holds

$$
\begin{aligned}
e^{\lambda t} \int_{\xi(t)}^{1}|\varphi(x)||u(t x)| \mathrm{d} x & \leq \int_{\xi(t)}^{1}|\varphi(x)| e^{\lambda t(1-x)} \mathrm{d} x \max _{t \in\left[T^{*}(|\mu|), T\right]} e^{\lambda t}|u(t)| \\
& \leq\left(e^{\lambda T^{*}(|\mu|) \delta} \Phi(1-\delta)+(\Phi(1)-\Phi(1-\delta))\right) \max _{t \in\left[T^{*}(|\mu|), T\right]} e^{\lambda t}|u(t)| .
\end{aligned}
$$

Hence (6) yields for sufficiently small $\delta>0$ and sufficiently large $\lambda<0$,

$$
|\mu| \max _{t \in\left[T^{*}(|\mu|), T\right]} e^{\lambda t}|u(t)| \leq \frac{1}{2}|\mu| \max _{t \in\left[T^{*}(|\mu|), T\right]} e^{\lambda t}|u(t)| .
$$

This implies that $\|u\|_{\infty,\left[T^{*}(|\mu|), T\right]}=0$ and the proof is complete.

\section{Concluding Remarks}

In this paper, we consider CVIEs with a vanishing delay: 
1) a proportional delay,

2) a nonlinear vanishing delay $\theta(t)$.

The first case reduces to a classical CVIE with a core limited to a subinterval. Hence these results are trivial from [4] [5]. For case 2), we present the compactness of the operators, i.e., supp $\varphi \subseteq\left[0, \theta^{\prime}(0)\right]$. In subsequent work, we will investigate the spectrum, eigenvalues and eigenfunctions when $\Phi\left(\theta^{\prime}(0)\right)<\Phi(1)$ and also numerical methods for CVIEs with vanishing delays.

\section{References}

[1] Bartoshevich, M.A. (1975) On a Heat Conduction Problem. Inž.-Fiz. Ž., 28, 340 346. (In Russian)

[2] Bartoshevich, M.A. (1975) Expansion in One Orthogonal System of Watson Operators for Solving Heat Conduction Problems. Inž.-Fiz. Ž., 28, 516-522. (In Russian)

[3] Šub-Sizonenko, J.A. (1979) Inversion of an Integral Operator by the Method of Expansion with Respect to Orthogonal Watson Operators. Siberian Math. J., 20, 318 321. https://doi.org/10.1007/BF00970048

[4] Vainikko, G. (2009) Cordial Volterra Integral Equations 1. Numer. Funct. Anal. Optim., 30, 1145-1172. https://doi.org/10.1080/01630560903393188

[5] Yang, Z.W. (2015) Second-Kind Linear Volterra Integral Equations with Noncompact Operators. Numer. Funct. Anal. Optim., 36, 104-131. https://doi.org/10.1080/01630563.2014.951769

[6] Volterra, V. (1897) Sopra alcune questioni di inversione di integrali definite. Ann. Mat. Pura Appl., 25, 139-178. https://doi.org/10.1007/BF02580504

[7] Fox, L., Mayers, D.F., Ockendon, J.R. and Tayler, A.B. (1971) On a Functional Differential Equation. J. Inst. Math. Appl., 8, 271-307.

https://doi.org/10.1093/imamat/8.3.271

[8] Ockendon, J.R. and Tayler, A.B. (1971) The Dynamics of a Current Collection System for an Electric Locomotive. Proc. Roy. Soc. Edinburg Sect. A, 322, 447-468. https://doi.org/10.1098/rspa.1971.0078

[9] Derfel, G.A. (1990) Kato Problem for Functional Equations and Difference Schrödinger Operators. Operator Theory, 46, 319-321. https://doi.org/10.1007/978-3-0348-7306-2_31

[10] Waltham, P. (1974) Deterministic Threshold Models in the Theory of Epidemics. Lecture Notes in Biomath., Vol. 1, Springer-Verlag, Berlin-Heidelberg.

[11] Brunner, H. (2009) Current Work and Open Problems in the Numerical Analysis of Volterra Functional Equations with Vanishing Delays. Front. Math. China, 4, 3-22. https://doi.org/10.1007/s11464-009-0001-0

[12] Iserles, A. (1993) On the Generalized Pantograph Functional-Differential Equation. European J. Appl. Math., 4, 1-38. https://doi.org/10.1017/S0956792500000966

[13] Vainikko, G. (2010) Cordial Volterra Integral Equations 2. Numer. Funct. Anal. Optim., 31, 191-219. https://doi.org/10.1080/01630561003666234

[14] Rudin, W. (1971) Functional Analysis. McGraw-Hill, New York. 
Submit or recommend next manuscript to SCIRP and we will provide best service for you:

Accepting pre-submission inquiries through Email, Facebook, LinkedIn, Twitter, etc. A wide selection of journals (inclusive of 9 subjects, more than 200 journals)

Providing 24-hour high-quality service

User-friendly online submission system

Fair and swift peer-review system

Efficient typesetting and proofreading procedure

Display of the result of downloads and visits, as well as the number of cited articles Maximum dissemination of your research work

Submit your manuscript at: http://papersubmission.scirp.org/

Or contact jamp@scirp.org 